\title{
Modeling and Simulation of Two-Phase Two-Component Flow with Disappearing Nonwetting Phase
}

\author{
Rebecca Neumann Peter Bastian Olaf Ippisch \\ Interdisciplinary Center for Scientific Computing \\ University of Heidelberg, Germany \\ Email: rebecca.neumann@iwr.uni-heidelberg.de
}

\begin{abstract}
Carbon Capture and Storage (CCS) is a recently discussed new technology, aimed at allowing an ongoing use of fossil fuels while preventing the produced $\mathrm{CO}_{2}$ to be released to the atmosphere. CSS can be modeled with two components (water and $\mathrm{CO}_{2}$ ) in two phases (liquid and $\mathrm{CO}_{2}$ ). To simulate the process, a multiphase flow equation with equilibrium phase exchange is used. One of the big problems arising in two-phase twocomponent flow simulations is the disappearance of the nonwetting phase, which leads to a degeneration of the equations satisfied by the saturation. A standard choice of primary variables, which is the pressure of one phase and the saturation of the other phase, cannot be applied here.

We developed a new approach using the pressure of the nonwetting phase and the capillary pressure as primary variables. One important advantage of this approach is the fact that we have only one set of primary variables that can be used for the biphasic as well as the monophasic case. We implemented this new choice of primary variables in the DUNE simulation framework and present numerical results for some test cases.
\end{abstract}

\section{Introduction}

In this work we address the mathematical modeling and numerical simulation of multiphase multicomponent flow in porous media with a special regard to $\mathrm{CO}_{2}$ storage in geologic formations. Some people consider $\mathrm{CO}_{2}$ storage, e.g. in deep saline aquifers, as an important factor in the effort to reduce the emission of greenhouse gases. Reliable simulation data is crucial for all stages of CCS projects.
After the $\mathrm{CO}_{2}$ injection, several different trapping mechanisms lead to an entrapment of the $\mathrm{CO}_{2}$. Shortly after the injection, structural trapping through caprocks is the most important factor. Later solubility trapping, where $\mathrm{CO}_{2}$ is dissolved into water, and residual trapping get more important. After several thousand years, there could also occur mineral trapping caused by geochemical reactions, but these are not considered in this work.

The mathematical model describing $\mathrm{CO}_{2}$ injection in geologic reservoirs is a two-phase two-component flow in porous media, a system of coupled, nonlinear partial differential equations. We do not only have two different phases (liquid and $\mathrm{CO}_{2}$ ), but also two components (water and $\mathrm{CO}_{2}$ ) in each phase, as the solubility of the components in the phases has to be taken into account. For an isothermal system we have to choose two primary variables and additional algebraic relations to close the system.

A standard choice for the primary variables is the pressure of one phase and the saturation of the other phase. A great challenge in this context is the disappearance of the nonwetting phase, which has been studied in many recent papers, as the saturations cannot be used as primary variable here. A valid choice in the one-phase region would be one phase pressure and the solubility of $\mathrm{CO}_{2}$ in the liquid phase.

Several Approaches to treat this problem exist, Class et al. (2002) switch primary variables depending on present phases, Jaffré et al. (2010) use complementarity conditions and Abadpour et al. (2009) extend the saturation to negative values. Bourgeat et al. (2010) use liquid phase pressure and water mass concentration as primary variables.

In this study we present a new choice of primary 
variables that is valid for the monophasic as well as the biphasic case and can easily handle the disappearance of one phase. One advantage of our approach is, that the chosen variables are continuous over material heterogeneities, if both phases are present.

To simulate CSS, constitutive relations between physical properties like pressure and density are necessary. We summarize our choice of existing approaches.

Numerical simulations for different test cases presented in this work will show that this new approach handles various applications very well. We use our new approach to simulate $\mathrm{CO}_{2}$ injection into the subsurface. A recent benchmark from the MoMas group concentrates on test cases arising from underground radioactive waste repository simulations. With our new set of primary variables we can also solve these problems efficiently.

\section{Mathematical model of a isothermal two-phase two-component flow}

In this section we will consider a porous medium and derive a system of partial differential equations describing two-phase two-component flow. For the sake of simplicity we use a constant temperature in this article, but thermodynamic effects can be included into the model in a straightforward manner. We also assume that the salinity of the water is constant.

\subsection{Notation}

We have two phases $\alpha \in\{\mathrm{w}, \mathrm{n}\}$, wetting and nonwetting, and two components $\kappa \in\{\mathrm{a}, \mathrm{b}\}$, water and nonwetting component.

$p_{\mathrm{w}}, p_{\mathrm{n}} \quad$ wetting and nonw. phase pressures $S_{\mathrm{w}}, S_{\mathrm{n}} \quad$ wetting and nonw. phase saturations $\rho_{\text {mass }, \mathrm{w}}, \rho_{\text {mass,n }}$ wetting and nonw. phase mass dens. $\rho_{\mathrm{mol}, \mathrm{w}}, \rho_{\mathrm{mol}, \mathrm{n}}$ wetting and nonw. phase molar dens. $\mu_{\mathrm{w}}, \mu_{\mathrm{n}}$ $x_{\mathrm{w}}^{\mathrm{a}}, x_{\mathrm{w}}^{\mathrm{b}}$ $x_{\mathrm{n}}^{\mathrm{a}}, x_{\mathrm{n}}^{\mathrm{b}}$ $M^{\mathrm{a}}, M^{\mathrm{b}}$ wetting and nonw. phase viscosities molar fraction of comp. in wet. phase molar fraction of comp. in nonw. phase molar mass of wet. and nonw. comp.

\subsection{Darcy's law}

The phase velocities $u_{\alpha}$ are given by an extended Darcy's Law:

$$
\begin{aligned}
& u_{\mathrm{w}}=-K \frac{k_{\mathrm{rw}}\left(S_{\mathrm{w}}\right)}{\mu_{\mathrm{w}}}\left(\nabla p_{\mathrm{w}}-\rho_{\mathrm{mass}, \mathrm{w}} \cdot g\right), \\
& u_{\mathrm{n}}=-K \frac{k_{\mathrm{rn}}\left(S_{\mathrm{n}}\right)}{\mu_{\mathrm{n}}}\left(\nabla p_{\mathrm{n}}-\rho_{\mathrm{mass}, \mathrm{n}} \cdot g\right),
\end{aligned}
$$

where $K$ is the absolute permeability, $k_{\text {rw }}$ and $k_{\text {rn }}$ denote the relative permeability functions and $g$ is the gravity vector.

The phase saturations and molar fractions satisfy

$$
S_{\mathrm{n}}+S_{\mathrm{w}}=1, \quad x_{\mathrm{w}}^{\mathrm{a}}+x_{\mathrm{w}}^{\mathrm{b}}=1, \quad x_{\mathrm{n}}^{\mathrm{a}}+x_{\mathrm{n}}^{\mathrm{b}}=1 .
$$

The relation between the phase pressures is given through the capillary pressure by the Brooks-Corey or van Genuchten-Mualem model

$$
p_{\mathrm{c}}\left(S_{\mathrm{w}}\right)=p_{\mathrm{n}}-p_{\mathrm{w}} .
$$

\subsection{Diffusive flux}

Following Fick's Law, the diffusive flux of a component $\kappa$ in the phase $\alpha$ is given by

$$
j_{\alpha}^{\kappa}=-D_{\mathrm{pm}, \alpha}^{\kappa} \rho_{\mathrm{mol}, \alpha} \nabla x_{\alpha}^{\kappa},
$$

where $D_{\mathrm{pm}, \alpha}^{\kappa}$ is the diffusion coefficient of component $\kappa$ in phase $\alpha$ in a porous medium.

Like 1] and 2] we assume

$$
j_{\alpha}^{\mathrm{a}}+j_{\alpha}^{\mathrm{b}}=0
$$

holds for simplicity, so we only need two diffusion coefficients instead of four.

\subsection{Mass conservation}

Local equilibrium phase exchange of the components in the phases is assumed. Taking into account the conservation of the amount of substance of each component and using (1), 22 and (5) we get the following partial differential equations describing an isothermal two-phase two-component flow:

$$
\begin{aligned}
\phi \partial_{t}\left\{\rho_{\mathrm{mol}, \mathrm{w}} x_{\mathrm{w}}^{\mathrm{a}} S_{\mathrm{w}}\right. & \left.+\rho_{\mathrm{mol}, \mathrm{n}} x_{\mathrm{n}}^{\mathrm{a}} S_{\mathrm{n}}\right\} \\
& +\nabla \cdot\left\{\rho_{\mathrm{mol}, \mathrm{w}} x_{\mathrm{w}}^{\mathrm{a}} u_{\mathrm{w}}+\rho_{\mathrm{mol}, \mathrm{n}} x_{\mathrm{n}}^{\mathrm{a}} u_{\mathrm{n}}\right\} \\
& +\nabla \cdot\left\{j_{\mathrm{w}}^{\mathrm{a}}+j_{\mathrm{n}}^{\mathrm{a}}\right\} \quad-q^{\mathrm{a}}=0 \\
\phi \partial_{t}\left\{\rho_{\mathrm{mol}, \mathrm{w}} x_{\mathrm{w}}^{\mathrm{b}} S_{\mathrm{w}}\right. & \left.+\rho_{\mathrm{mol}, \mathrm{n}} x_{\mathrm{n}}^{\mathrm{b}} S_{\mathrm{n}}\right\} \\
& +\nabla \cdot\left\{\rho_{\mathrm{mol}, \mathrm{w}} x_{\mathrm{w}}^{\mathrm{b}} u_{\mathrm{w}}+\rho_{\mathrm{mol}, \mathrm{n}} x_{\mathrm{n}}^{\mathrm{b}} u_{\mathrm{n}}\right\} \\
& +\nabla \cdot\left\{j_{\mathrm{w}}^{\mathrm{b}}+j_{\mathrm{n}}^{\mathrm{b}}\right\} \quad-q^{\mathrm{b}}=0,
\end{aligned}
$$

where $q^{\mathrm{a}}$ and $q^{\mathrm{b}}$ are the source/sink terms for the components.

\section{Constitutive Relations}

We will now look at the special case of CCS where a liquid water phase and a liquid, gaseous or supercritical $\mathrm{CO}_{2}$ phase are present. The components are water and $\mathrm{CO}_{2}$. In the following the different functions and Equations of State (EOS) to determine secondary parameters are described. Additionally their dependence on other variables is given. 


\subsection{Solubility of components}

The solubility of the components is influenced by the pressure $p_{\mathrm{n}}$ and the temperature $T$ of the system, the salinity $s_{\text {sal }}$ of water also plays an important role:

$$
x_{\mathrm{w}}^{\mathrm{b}}\left(p_{\mathrm{n}}, T, s_{\mathrm{sal}}\right), \quad x_{\mathrm{n}}^{\mathrm{a}}\left(p_{\mathrm{n}}, T, s_{\mathrm{sal}}\right) .
$$

There exist different EOS for this system. We use the EOS by Spycher \& Pruess [3], because in contrast to other models (for example, the EOS of Duan \& Sun [4) also the solubility of water in $\mathrm{CO}_{2}$ is described very well. Figure 1 and 2 show the solubility curves for different temperatures.

The solubility of $\mathrm{CO}_{2}$ in the water phase increases fast with rising pressure up to the saturation pressure, above that it rises with a smaller rate. For temperatures below the critical temperature $T_{\text {crit }}=304.15 \mathrm{~K}$, the state of the carbon dioxide changes from gaseous (below saturation pressure) to liquid which results in a not continuously differentiable sharp break at the transition point.

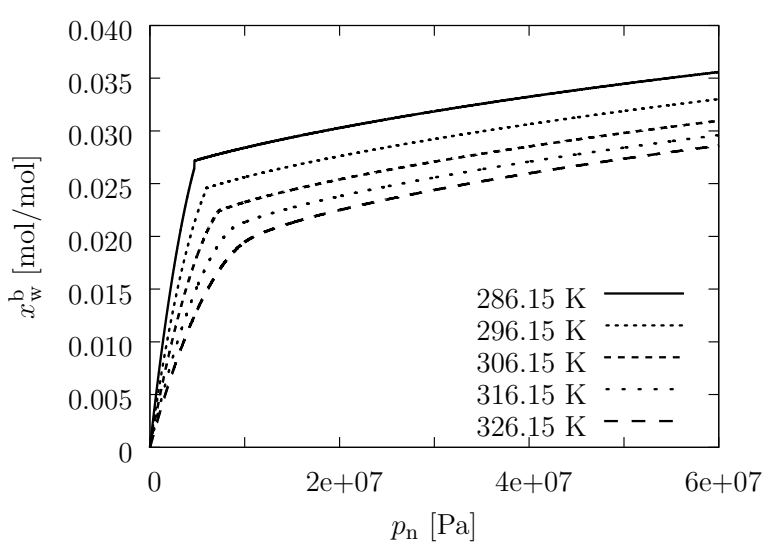

Figure 1: Solubility $x_{\mathrm{w}}^{\mathrm{b}}$ for different temperatures

$$
\left(s_{\text {sal }}=0\right)
$$

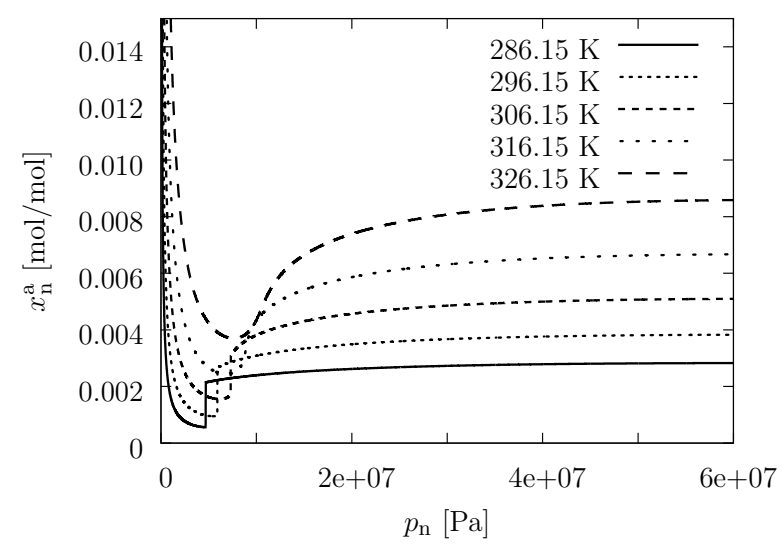

Figure 2: Solubility $x_{\mathrm{n}}^{\mathrm{a}}$ for different temperatures $\left(s_{\mathrm{sal}}=0\right)$

\subsection{Densities}

For the density of the water phase the approach of Garcia [5] is applied. The density increases slightly for a larger fraction of $\mathrm{CO}_{2}$ in the water phase. The EOS of Duan [6 is used to calculate the density of the $\mathrm{CO}_{2}$ phase, which strongly depends on the $\mathrm{CO}_{2}$ phase pressure,

$$
\rho_{\text {mass }, \mathrm{w}}\left(x_{\mathrm{w}}^{\mathrm{b}}, T\right), \quad \rho_{\text {mass }, \mathrm{n}}\left(p_{\mathrm{n}}, T\right) .
$$

Figure 3 shows the density of $\mathrm{CO}_{2}$ for different temperatures. To convert mass density to molar density the phase composition has to be taken into account,

$$
\rho_{\mathrm{mol}, \alpha}=\frac{\rho_{\mathrm{mass}, \alpha}}{x_{\alpha}^{\mathrm{b}} M^{\mathrm{b}}+x_{\alpha}^{\mathrm{a}} M^{\mathrm{a}}} .
$$

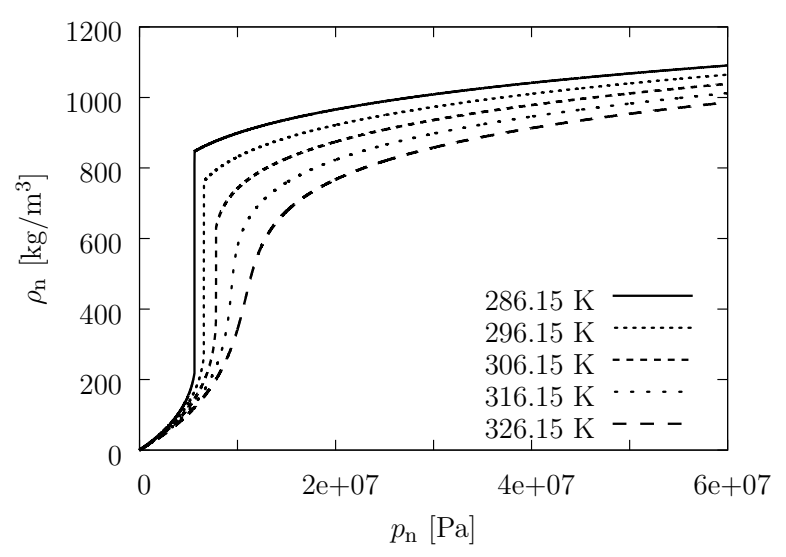

Figure 3: $\mathrm{CO}_{2}$ density for different temperatures

\subsection{Viscosities}

The viscosity of the water phase is computed with a function from Atkins [7, for the $\mathrm{CO}_{2}$ phase we use the approach of Fenghour \& Vesovic [8]. Again the $\mathrm{CO}_{2}$ phase viscosity strongly depends on the $\mathrm{CO}_{2}$ phase pressure,

$$
\mu_{\mathrm{w}}(T), \quad \mu_{\mathrm{n}}\left(p_{\mathrm{n}}, T\right)
$$

Figure 4 shows the viscosity of $\mathrm{CO}_{2}$ for different temperatures.

\subsection{Diffusion}

Following [9], we use an approach suggested by Millington \& Quirk

$$
D_{\mathrm{pm}, \alpha}^{\kappa}=\frac{\left(\phi S_{\alpha}\right)^{10 / 3}}{\phi^{2}} D_{\alpha}^{\kappa},
$$

for the diffusion coefficient in the porous medium, where $D_{\alpha}^{\kappa}$ describes the binary diffusion coefficient of component $\kappa$ in phase $\alpha$. 


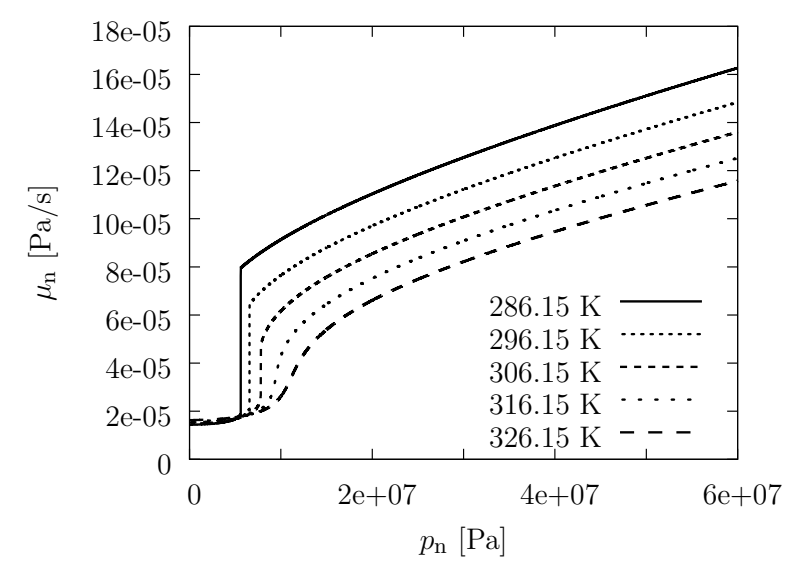

Figure 4: $\mathrm{CO}_{2}$ viscosity for different temperatures

\section{Choice of primary variables}

A standard choice for the primary variables are one phase pressure and the saturation. In the one phase region $\left(S_{\mathrm{n}}=0\right)$, the system $(7)$ degenerates to

$$
\begin{gathered}
\phi \partial_{t}\left\{\rho_{\mathrm{mol}, \mathrm{w}} x_{\mathrm{w}}^{\mathrm{a}}\right\}+\nabla \cdot\left\{\rho_{\mathrm{mol}, \mathrm{w}} x_{\mathrm{w}}^{\mathrm{a}} u_{\mathrm{w}}+j_{\mathrm{w}}^{\mathrm{a}}\right\}-q^{\mathrm{a}}=0, \\
\phi \partial_{t}\left\{\rho_{\mathrm{mol}, \mathrm{w}} x_{\mathrm{w}}^{\mathrm{b}}\right\}+\nabla \cdot\left\{\rho_{\mathrm{mol}, \mathrm{w}} x_{\mathrm{w}}^{\mathrm{b}} u_{\mathrm{w}}+j_{\mathrm{w}}^{\mathrm{b}}\right\}-q^{\mathrm{b}}=0 .
\end{gathered}
$$

Using (3) and (6) the system can be rewritten as a coupled groundwater-flow and transport problem

$$
\begin{aligned}
& \phi \partial_{t}\left\{\rho_{\mathrm{mol}, \mathrm{w}}\right\}+\nabla \cdot\left\{\rho_{\mathrm{mol}, \mathrm{w}} u_{\mathrm{w}}\right\}=q^{\mathrm{a}}+\quad q^{\mathrm{b}}, \\
& \phi \partial_{t}\left\{\rho_{\mathrm{mol}, \mathrm{w}} x_{\mathrm{w}}^{\mathrm{b}}\right\}+\nabla \cdot\left\{\rho_{\mathrm{mol}, \mathrm{w}} x_{\mathrm{w}}^{\mathrm{b}} u_{\mathrm{w}}+j_{\mathrm{w}}^{\mathrm{b}}\right\}=q^{\mathrm{b}} .
\end{aligned}
$$

With the disappearance of the nonwetting phase the saturation can no longer be used as primary variable and the standard choice of variables cannot be applied here. One natural set of variables for the one phase region would be $p_{\mathrm{w}}$ and $x_{\mathrm{w}}^{\mathrm{b}}$. There are several approaches

\begin{tabular}{lr}
\hline Primary Var. & Method \\
\hline$S_{\mathrm{n}}, p_{\mathrm{n}}$ & $\begin{array}{r}\text { Extending the saturation } \\
\text { to negative values (see [10). }\end{array}$ \\
$S_{\mathrm{w}}, p_{\mathrm{w}}, X_{\mathrm{w}}^{\mathrm{b}}$ & $\begin{array}{r}\text { Using complementarity constraints } \\
(\text { see [11]). }\end{array}$ \\
$p_{\mathrm{n}},\left(S_{\mathrm{w}}\right.$ or $\left.X_{\mathrm{w}}^{\mathrm{b}}\right)$ & $\begin{array}{r}\text { Switching primary variables depen- } \\
\text { ding on present phases (see [12], [13]). }\end{array}$ \\
\hline
\end{tabular}

Table 1: Several methods to deal with a disappearing nonwetting phase

to solve the problem at the phase transition (see Table 1. $X_{\alpha}^{\kappa}$ denotes the mass fraction).

A common method is primary variable switching used for example by Forsyth \& Simpson 12 and Helmig \& Class [13. Here different sets of primary variables are used in the one phase and two phase region, the variables are switched if a phase appears or disappears.

Abadpour \& Panfilov [10] extend the saturation to artificial negative values, so that system (7) does not degenerate in the one phase region and the saturation can still be used as a primary variable.

Jaffré \& Sboui [11] use the solubility as an additional third primary variable. Additional nonlinear complementarity constraints, which describe the transition from one phase to two phase region are used to close the system.

We developed a new approach using the pressure of the nonwetting phase and the capillary pressure as primary variables. In the absence of the nonwetting phase, $p_{\mathrm{n}}$ is defined as the corresponding pressure to the solubility $x_{\mathrm{w}}^{\mathrm{b}}$.

Our approach has the advantage, that we only have two primary variables in contrast to the complementarity constraints method, where an additional variable is needed. With our constant set of variables we also avoid a switching of the primary variables, which is a non-differentiable process that can lead to numerical difficulties.

This idea was first presented by Ippisch [14. In the context of nuclear waste management for the special case that Henry's Law is used to couple solubility and pressure there exist similar approaches. Bourgeat et al. [2] use the water mass concentration and the wetting phase pressure, Angelini et al. [15] use the two phase pressures as primary variables.

In section 5 we will apply our approach not only to a recent benchmark study on nuclear waste management, but also to the very challenging field of CCS. In contrast to nuclear waste management and the work of Bourgeat et al. and Angelini et al. it is not possible to use Henry's Law for the solubility, because the approximation is not valid for $\mathrm{CO}_{2}$. We need a nonlinear function (see subsection 3.1 to describe the dependency between the nonwetting pressure and mole fraction. Moreover we have to handle the very high injection rate of the $\mathrm{CO}_{2}$.

\section{$4.1 p_{\mathrm{n}} / p_{c}$ formulation: Interpretation as algebraic transformation}

The entry pressure $p_{\text {entry }}$ is the critical capillary pressure that must be applied so that the nonwetting phase appears. We have to distinguish between

1. $p_{\mathrm{c}} \leq p_{\text {entry }}$ where $S_{\mathrm{n}}=0$ and only the wetting phase exists

2. $p_{\mathrm{c}}>p_{\text {entry }}$ where $S_{\mathrm{n}}>0$ and both wetting and nonwetting phase exist. 
Case 1: $p_{\mathrm{c}} \leq p_{\text {entry }}$

As mentioned in the beginning of the section the natural set of variables for the one phase system (9) would be $p_{\mathrm{w}}$ and $x_{\mathrm{w}}^{\mathrm{b}}$. Consider the following transformation of variables

$$
\begin{aligned}
& p_{\mathrm{w}}=p_{\mathrm{n}}-p_{\mathrm{c}} \\
& x_{\mathrm{w}}^{\mathrm{b}}=\psi\left(p_{\mathrm{n}}\right)
\end{aligned}
$$

where $\psi$ is a continuous and invertible function. The solubility relation for $x_{\mathrm{w}}^{\mathrm{b}}$ (8) satisfies these demands (see Figure 11 and Spycher \& Pruess [3]). The mapping between $p_{\mathrm{n}}$ and $x_{\mathrm{w}}^{\mathrm{b}}$ is hence unique and $p_{\mathrm{n}}$ and $p_{\mathrm{c}}$ is a valid set of primary variables.

The relation between the capillary pressure and the saturation $p_{\mathrm{c}}\left(S_{\mathrm{w}}\right)$ (see Equation (3p) is a strictly decreasing function for $S_{\mathrm{w}} \in[0,1]$ and can therefore be inverted

$$
S_{\mathrm{w}}=\eta\left(p_{\mathrm{c}}\right) .
$$

The dependent variables are then obtained through

$$
\begin{aligned}
& S_{\mathrm{w}}=\eta\left(p_{\mathrm{c}}\right) \quad S_{\mathrm{n}}=1-\eta\left(p_{\mathrm{c}}\right) \\
& x_{\mathrm{w}}^{\mathrm{b}}=\psi\left(p_{\mathrm{n}}\right) \quad x_{\mathrm{w}}^{\mathrm{a}}=1-\psi\left(p_{\mathrm{n}}\right) \\
& x_{\mathrm{n}}^{\mathrm{a}}=\gamma\left(p_{\mathrm{n}}\right) \quad x_{\mathrm{n}}^{\mathrm{b}}=1-\gamma\left(p_{\mathrm{n}}\right)
\end{aligned}
$$

where $\gamma$ is the solubility curve given in (8). All other variables are computed as given in Section 3 .

This choice is not unique, another possible set would be $p_{\mathrm{w}} / p_{c}$ or $p_{\mathrm{w}} / p_{\mathrm{n}}$. Using $p_{\mathrm{n}}$ as a primary variable has the advantage, that the highly nonlinear density and viscosity functions are directly dependent on a primary variable. We prefer $p_{c}$ over $p_{l}$ as additional primary variable, because then the saturation only depends on the primary variable $p_{c}$ through the nonlinear capillary pressure-saturation relationship.

Instead of the nonwetting phase pressure $p_{\mathrm{n}}$ the molar fraction $x_{\mathrm{w}}^{\mathrm{b}}=\psi\left(p_{\mathrm{n}}\right)$ could also be used as primary variable, which is very similar to the water mass concentration used by Bourgeat et al. 2].

\section{Case 2: $p_{\mathrm{c}}>p_{\text {entry }}$}

The common choice of primary variables in the twophase region is one pressure and the saturation. With the $p_{\mathrm{n}} / p_{\mathrm{c}}$ formulation we obtain the saturations through the retention curve $S_{\mathrm{w}}=\eta\left(p_{\mathrm{c}}\right)$, the other variables are computed accordingly.

$p_{\mathrm{w}}$ and $x_{\mathrm{w}}^{\mathrm{b}}$ are continuous at the interface between the one-phase and the two-phase region. Through the transformation 10, $p_{\mathrm{n}}$ and $p_{\mathrm{c}}$ are continuous at the interface too. With $p_{\mathrm{n}} / p_{\mathrm{c}}$ we found a set of primary variables that can be consistently used in the presence or absence of the nonwetting phase. One advantage of the $p_{\mathrm{n}} / p_{\mathrm{c}}$ formulation is, that the pressures, in contrast to the saturations, are continuous across material heterogeneities if both phases exist.

\section{Numerical simulation}

In the following section we present the numerical results for special test cases. All simulations were performed in the DUNE simulation framework [16, 17].

A cell-centered finite volume method with two-point flux approximation on a structured grid was used for the domain discretization. The grid $E_{h}=e_{1}, \ldots, e_{n}$ consists of elements $e_{i}$ and the boundary of each element is $\partial e_{i}=\bigcup_{j \in \Sigma(i)} \gamma_{i j}$ where $\gamma_{i j}$ denotes the boundary between elements $e_{i}$ and $e_{j}$. The cell-centered finite volume method for Equation (7) for each component $\kappa$ then reads

$$
\begin{aligned}
& \sum_{e_{i} \in E_{h}}\left\{\int_{e_{i}} \phi \partial_{t}\left\{\rho_{\mathrm{mol}, \mathrm{w}} x_{\mathrm{w}}^{\kappa} S_{\mathrm{w}}+\rho_{\mathrm{mol}, \mathrm{n}} x_{\mathrm{n}}^{\kappa} S_{\mathrm{n}}\right\} \mathrm{d} e\right. \\
& +\frac{1}{\left\|e_{i}\right\|} \sum_{j \in \Sigma(i)} \int_{\gamma_{i j}}\left(\nabla \cdot\left\{\rho_{\mathrm{mol}, \mathrm{w}} x_{\mathrm{w}}^{\kappa} u_{\mathrm{w}}+\rho_{\mathrm{mol}, \mathrm{n}} x_{\mathrm{n}}^{\kappa} u_{\mathrm{n}}\right\}\right. \\
& \left.\left.+\nabla \cdot\left\{j_{\mathrm{w}}^{\kappa}+j_{\mathrm{n}}^{\kappa}\right\}-q^{\kappa}\right) \cdot n_{i j} \mathrm{~d} \gamma\right\}=0
\end{aligned}
$$

where $n_{i j}$ denotes the unit outer normal to $\gamma_{i j}$.

A special upwinding scheme is used to calculate the phase fluxes at the interface between two elements to handle material discontinuities resulting in different capillary-pressure saturation curves and relative permeability functions in both elements.

The direction of the flux of phase $\alpha$ at the interface between two elements $i$ and $j$ can be obtained from the sum of the pressure gradient and the force of gravitation $w_{\alpha, i j}=-\left(\nabla p_{\alpha}-\rho_{\text {mass }, \alpha, i j} \cdot g\right) \cdot n_{i j}$, where $\rho_{\text {mass }, \alpha, i j}$ is computed as the arithmetic average of cells $e_{i}$ and $e_{j}$. Depending on the sign of $w_{\alpha, i j}$ the upwind element is determined.

$$
\text { upwind }_{\alpha}=\left\{\begin{array}{ll}
i & w_{\alpha, i j} \geq 0 \\
j & \text { else }
\end{array} .\right.
$$

The capillary pressure of the upwind element is used to calculate the relative permeability in each element. The obtained relative permeabilities are multiplied by the absolute permeabilities and the viscosities in each element. A harmonic average of the values is used to calculate the flux at the interface:

$$
\begin{aligned}
K_{\alpha, i} & =K_{i} \frac{k_{r \alpha, i}\left(p_{c, \text { upwind }_{\alpha}}\right)}{\mu_{\alpha, i}} \\
K_{\alpha, j} & =K_{j} \frac{k_{r \alpha, j}\left(p_{c, \text { upwind }}\right)}{\mu_{\alpha, j}} \\
u_{\alpha, i j} & =\frac{K_{\alpha, i} K_{\alpha, j}}{K_{\alpha, i}+K_{\alpha, j}} w_{\alpha, i j}
\end{aligned}
$$


For homogeneous porous media this upwinding scheme corresponds to an upwinding of saturation.

For the calculation of the convective component transport a full upwinding of the molar fractions and the molar densities based on the upwind direction is used with

$$
x_{\alpha, i j}^{\kappa}=x_{\alpha, \text { upwind }_{\alpha}}^{\kappa}, \quad \rho_{\text {mol }, \alpha, i j}=\rho_{\text {mol }, \alpha, \text { upwind }_{\alpha}} .
$$

As time discretization scheme the implicit Euler Method was used. Newton's Method was applied to linearize the system. The Jacobian matrix is derived through numerical differentiation. The resulting linear equation system is solved with a BiCGStab solver preconditioned by an algebraic mul-tigrid method (see [18]).

We chose three different test cases, the first one is from a recent benchmark study concentrating on appearance and disappearance of phases in the context of nuclear waste management. As there are no analytical solutions for two-phase two-component flow systems, we use the results of other groups as possibility to validate our results. We also conduct a grid convergence study to verify the experimental order of convergence of our implementation.

With the second test case we apply our formulation to a $\mathrm{CO}_{2}$ sequestration scenario in $2 \mathrm{D}$ and perform a strong scalability test. The third test case extends the second test case to 3D and shows that our approach can handle the large number of unknowns.

The simulations were performed in parallel with up to 16 processes.

\section{Test case 1: Gas injection in a fully water saturated domain (quasi-1D)}

The first test case is an example from the MoMas benchmark on multiphase flow in porous media 19, [20]. We converted the descriptions to match the variables used in this paper.

In this case the considered nonwetting component is hydrogen and the wetting component is water. The solubility of water in the nonwetting phase is neglected: $x_{\mathrm{n}}^{\mathrm{a}}=0$. Hydrogen is injected into the left part of a rectangular domain $(200 \mathrm{~m} \times 20 \mathrm{~m})$ with a flux of $q_{\mathrm{n}}^{\text {in }}$ for $5 \cdot 10^{5}$ years. The domain is initially fully saturated by the water phase, consisting only of pure water with initial conditions $p_{\alpha}=p_{\alpha}^{\mathrm{i}}$ (see Table 2). The boundary conditions are Neumann 0 boundaries at the top and bottom (see Figure 5). The Dirichlet boundary conditions for the outflow boundary are the same as the initial conditions: $\left.p_{\alpha}\right|_{\Gamma_{\text {out }}}=p_{\alpha}^{\mathrm{i}}$. Gravitation is neglected, which leads to a quasi-1D problem.

The relationship between $p_{\mathrm{n}}$ and $X_{\mathrm{w}}^{\mathrm{b}}$ (where $X_{\alpha}^{\kappa}$ is the mass fraction in contrast to the molar fraction $x_{\alpha}^{\kappa}$ )

\begin{tabular}{|c|c|c|c|c|}
\hline \multicolumn{3}{|c|}{ Value } & \multicolumn{2}{|l|}{ Value } \\
\hline$\phi$ & 0.15 & & $q^{\kappa} \quad 0$ & \\
\hline$S_{\mathrm{w}, \mathrm{res}}$ & 0.4 & & $q_{\mathrm{n}}^{\mathrm{in}} 1.77 \cdot 10^{-13}$ & $\mathrm{~kg} \mathrm{~m}^{-2} \mathrm{~s}^{-1}$ \\
\hline$S_{\mathrm{n}, \mathrm{res}}$ & 0 & & $D_{\mathrm{w}}^{\mathrm{b}} 3 \quad \cdot 10^{-9}$ & $\mathrm{~m}^{2} \mathrm{~s}^{-1}$ \\
\hline$n$ & 1.49 & & $D_{\mathrm{n}}^{\mathrm{a}} 0$ & $\mathrm{~m}^{2} \mathrm{~s}^{-1}$ \\
\hline$\alpha$ & $5 \cdot 10^{-7}$ & $\mathrm{~Pa}^{-1}$ & $\cdot 10^{-3}$ & $\mathrm{Pas}^{-1}$ \\
\hline$M^{\mathrm{a}}$ & $\cdot 10^{-2}$ & $\mathrm{~kg} \mathrm{~mol}^{-1}$ & $\mu_{\mathrm{n}} 9 \cdot 10^{-6}$ & $\mathrm{Pas}^{-1}$ \\
\hline$M^{\mathrm{b}}$ & $\cdot 10^{-3}$ & $\mathrm{~kg} \mathrm{~mol}^{-1}$ & $7.65 \cdot 10^{-6}$ & $\mathrm{~mol} \mathrm{~m}{ }^{-3} \mathrm{~Pa}^{-1}$ \\
\hline$p_{\mathrm{w}}^{\mathrm{i}}$ & $\cdot 10^{6}$ & $\mathrm{~Pa}$ & $5 \cdot 10^{-20}$ & $\mathrm{~m}^{2}$ \\
\hline$p_{\mathrm{n}}^{\mathrm{i}}$ & 0 & $\mathrm{~Pa}$ & & \\
\hline
\end{tabular}

Table 2: Parameters for test case 1

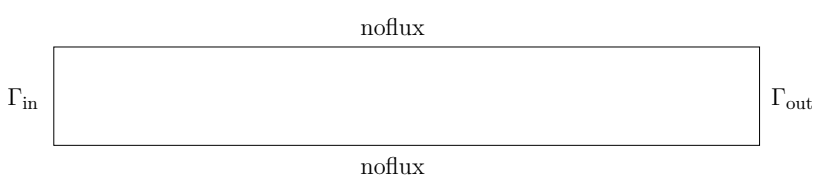

Figure 5: Test case 1: Domain setup

is given through Henry's Law:

$$
X_{\mathrm{w}}^{\mathrm{b}}=\frac{H(T)}{\rho_{\mathrm{mol}, \mathrm{w}}} p_{\mathrm{n}}^{\mathrm{b}},
$$

where the partial pressure of hydrogen in the nonwetting phase is $p_{\mathrm{n}}^{\mathrm{b}}=p_{\mathrm{n}}$ for this case because there is no water in the nonwetting phase for this example. The mass fraction $X_{\mathrm{w}}^{\mathrm{b}}$ is then converted to the molar fraction:

$$
x_{\mathrm{w}}^{\mathrm{b}}=\frac{X_{\mathrm{w}}^{\mathrm{b}} M^{\mathrm{a}}}{X_{\mathrm{w}}^{\mathrm{b}} M^{\mathrm{a}}+\left(1-X_{\mathrm{w}}^{\mathrm{b}}\right) M^{\mathrm{b}}} .
$$

The nonwetting phase density is determined by the ideal gas law, wetting phase density is obtained through Henry's Law

$\rho_{\text {mass }, \mathrm{n}}=p_{\mathrm{n}} M^{\mathrm{b}}(R T)^{-1}, \quad \rho_{\text {mass }, \mathrm{w}}=10^{3}+H(T) M^{\mathrm{n}} p_{\mathrm{b}}$.

The diffusion coefficient is given as

$$
D_{\mathrm{pm}, \alpha}^{\kappa}=\phi S_{\alpha}\left(\frac{X_{\alpha}^{\mathrm{n}}}{M^{\mathrm{n}}}+\frac{X_{\alpha}^{\mathrm{a}}}{M^{\mathrm{a}}}\right) D_{\alpha}^{\kappa} .
$$

A van Genuchten-Mualem model with the parameters $n, \alpha$ and $S_{\alpha, \text { res }}$ as given in Table 2 is used for the soil water characteristic and relative permeabilities. All other parameters used in the simulation are also noted in Table 2 .

A structured grid with $400 \times 20$ cells was used for the computations. Figure 6 and 7 show the nonwetting phase saturation and phase pressures at $\Gamma_{\text {in }}$ over time. $S_{\mathrm{n}}$ is zero at the beginning, all injected hydrogen dissolves into the wetting phase and no nonwetting phase is present. At $t \approx 13000$ years a nonwetting phase starts to appear at the injection point $\Gamma_{\text {in }}$. 


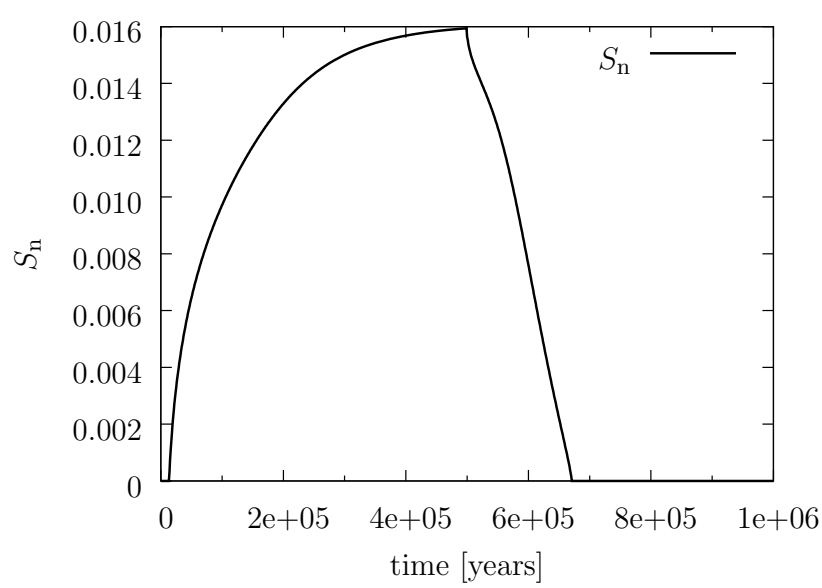

Figure 6: Test case 1: Nonwetting phase saturation at $\Gamma_{\text {in }}$

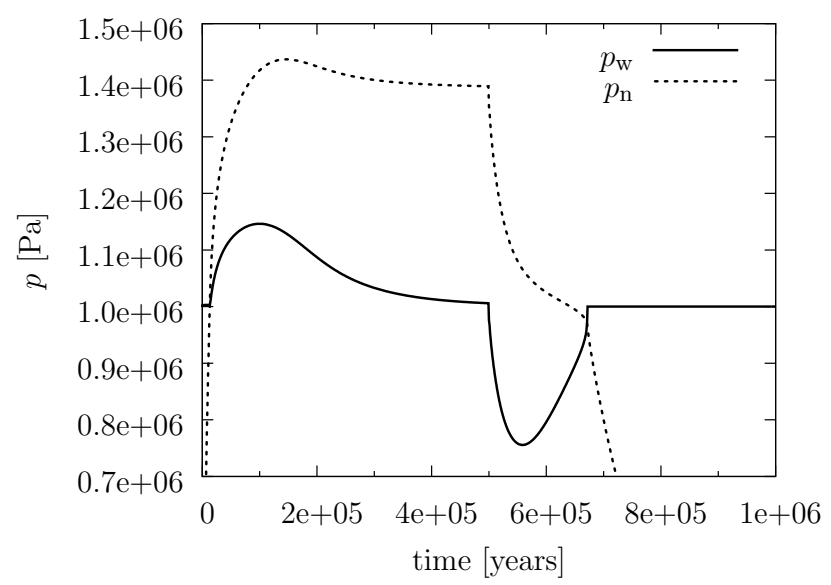

Figure 7: Test case 1: Phase pressures at $\Gamma_{\text {in }}$

For the computations we used a constant time step $d t=1000$ years. We also verified the robustness of our model by using larger time steps ( $d t=5000$ years).

Six different groups including our group participated in this benchmark example, the results of all groups are presented in [20]. The results of our simulation corresponds well to the results of the other groups.

In addition we performed a grid convergence study. For the coarsest level (level 1) we use $12 \times 1$ cells. For each level we double the amount of grid cells in $x$-direction, so we have $12 \times 2^{i}$ cells for level $i$. The solution on level 12 was used as a reference solution. The resulting experimental order of convergence (EOC) can then be computed through

$$
\mathrm{EOC}_{i+1}=\frac{1}{\log (2)}\left|\log \left(\frac{e_{i}}{e_{i+1}}\right)\right|
$$

where $e_{i}$ is the $L 2$-error between the solution on level $i$ and the reference solution. At $t=2 \cdot 10^{5}$ years we get second order grid convergence for nonwetting phase pressure and capillary pressure (see Table 3 ).

\begin{tabular}{rrrr}
\hline level & \#elements & EOC $\left(p_{c}\right)$ & EOC $\left(p_{\mathrm{n}}\right)$ \\
\hline 1 & 24 & 2.01 & 2.02 \\
2 & 48 & 1.97 & 1.98 \\
3 & 96 & 1.98 & 1.98 \\
4 & 192 & 1.99 & 1.99 \\
5 & 384 & 2.00 & 1.99 \\
6 & 768 & 2.00 & 2.00 \\
7 & 1536 & 2.01 & 2.01 \\
8 & 3072 & 2.03 & 2.02 \\
\hline
\end{tabular}

Table 3: Grid convergence study for test case 1

The convergence study shows that our numerical solution converges with an optimal EOC of two, which is the maximum order that can be achieved with a cellcentered finite volume discretization.

\section{Test case 2: $\mathrm{CO}_{2}$ injection in a fully water saturated domain (2D)}

In the second test case $\mathrm{CO}_{2}$ is injected into the lower left part of a rectangular geometry $(600 \mathrm{~m} \times 100 \mathrm{~m})$ with a flux of $q_{\mathrm{b}}^{\mathrm{in}}$. The domain is located $800 \mathrm{~m}$ under the surface. As in test case 1, the top and bottom of the domain have noflux boundary conditions (see Figure 9). For the Dirichlet boundary on the right side we

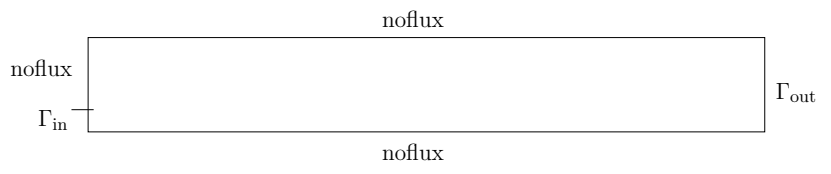

Figure 9: Test case 2: Domain setup

choose hydrostatic pressure for the water phase and zero pressure for the $\mathrm{CO}_{2}$ phase (which leads to $x_{\mathrm{w}}^{\mathrm{n}}=0$ )

$\left.p_{\mathrm{w}}\right|_{\Gamma_{\text {out }}}=10^{5}+(900-z) \rho_{\text {mass }, \mathrm{w}} \cdot g \mathrm{~Pa},\left.\quad p_{\mathrm{b}}\right|_{\Gamma_{\text {out }}}=0 \mathrm{~Pa}$,

where $z$ is the $z$-coordinate in the domain and $g$ the gravity in $z$-direction. Again the same values are taken as initial values.

\begin{tabular}{|c|c|c|c|c|c|}
\hline & \multicolumn{3}{|c|}{ Value } & \multicolumn{2}{|l|}{ Value } \\
\hline$\phi$ & 0.2 & & & $q^{\kappa} \quad 0$ & \\
\hline$S_{\alpha, \text { res }}$ & 0 & & & $q_{\mathrm{n}}^{\text {in }} 4 \cdot \cdot 10^{-2}$ & $\mathrm{~kg} \mathrm{~m}^{-2} \mathrm{~s}^{-1}$ \\
\hline$\lambda$ & 2 & & & $\cdot 10^{-9}$ & $\mathrm{~m}^{2} \mathrm{~s}^{-1}$ \\
\hline$p_{\text {entry }}$ & 1 & $\cdot 10^{3}$ & $\mathrm{~Pa}$ & $D_{\mathrm{n}}^{\mathrm{a}} 2 \cdot 10^{-9}$ & $\mathrm{~m}^{2} \mathrm{~s}^{-1}$ \\
\hline$K$ & 1 & $\cdot 10^{-12}$ & $\mathrm{~m}^{2}$ & $M^{\mathrm{a}} 1.8 \cdot 10^{-2}$ & $\mathrm{~kg} \mathrm{~mol}^{-1}$ \\
\hline$T$ & 313.15 & & $\mathrm{~K}$ & $M^{\mathrm{b}} 4.4 \cdot 10^{-2}$ & $\mathrm{~kg} \mathrm{~mol}^{-1}$ \\
\hline
\end{tabular}

Table 4: Parameters for test case 2

Densities, viscosities and solubilities are chosen as suggested in Section 3, all other parameters are given 


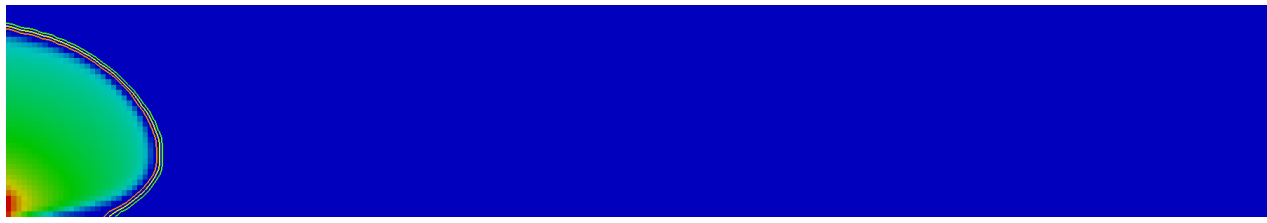

(a) 7 days, $\quad \max \left(S_{\mathrm{n}}\right)=0.82, \max \left(x_{\mathrm{w}}^{\mathrm{b}}\right)=0.022$

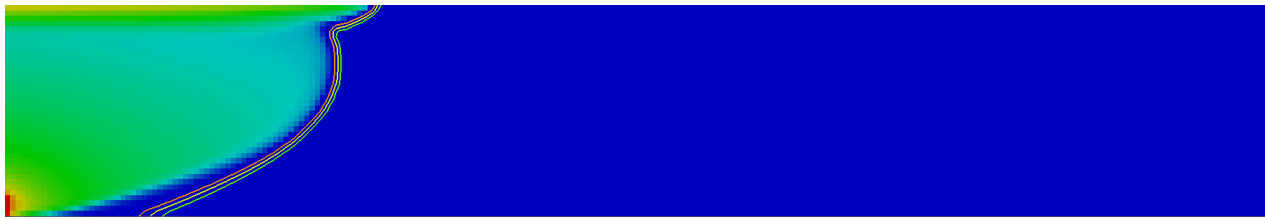

(b) 20 days, $\quad \max \left(S_{\mathrm{n}}\right)=1, \max \left(x_{\mathrm{w}}^{\mathrm{b}}\right)=0.020$

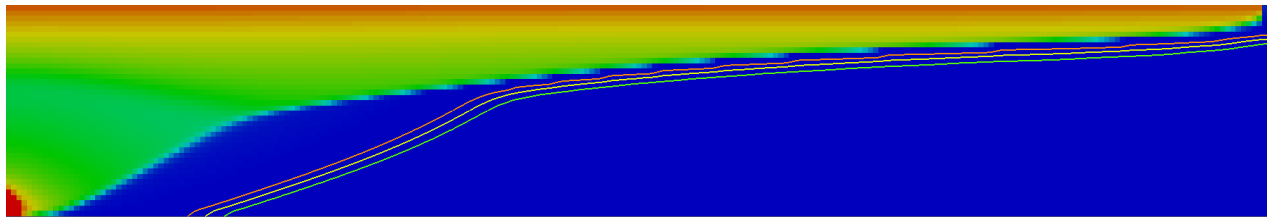

(c) 65 days, $\quad \max \left(S_{\mathrm{n}}\right)=1, \max \left(x_{\mathrm{w}}^{\mathrm{b}}\right)=0.020$

Figure 8: Test case 2: $\mathrm{CO}_{2}$ phase saturation and molar fraction of dissolved $\mathrm{CO}_{2}$ in water (contour lines for $\left.x_{\mathrm{w}}^{\mathrm{b}}=0.005,0.011,0.016\right)$. Color scale ranges from $S_{\mathrm{n}}=0$ (blue) to $S_{\mathrm{n}}=\max \left(S_{\mathrm{n}}\right)($ red) .

in Table 4. In this example we used the Brooks-Corey model for the soil water characteristic and relative permeabilities. For the computations a structured grid with $240 \times 40$ cells was used.

The results of test case 2 are shown in Figure 8, Each picture shows the $\mathrm{CO}_{2}$ phase saturation at a specific time point, the contour lines depict the molar fraction of $\mathrm{CO}_{2}$ in the water phase. The $\mathrm{CO}_{2}$ migrates upwards until it reaches the top of the domain with the noflux conditions and is then driven to the right by advective forces. Around the $\mathrm{CO}_{2}$ front the water phase contains dissolved $\mathrm{CO}_{2}$.

An analytical solution for this test case does not exist, but the simulation results are plausible and the $\mathrm{CO}_{2}$ front behaves as expected.

During the initial phase of CCS $\mathrm{CO}_{2}$ is injected and the $\mathrm{CO}_{2}$ front is propagating. Thus for the sake of accuracy we want to choose a time step size so that the $\mathrm{CO}_{2}$ front travels one grid cell layer per time step.

For the computations we used a maximum time step of $d t=5000 \mathrm{~s}$. The time step size is halved if the Newton solver did not converge, it is doubled until the maximum time step is reached in case of convergence. With this time step control we achieve an average time step size of $d t=3575 \mathrm{~s}$ and the $\mathrm{CO}_{2}$ front moves about one grid cell layer per time step, which fulfills the above condition.

All simulations were done in parallel. To analyze the parallel performance of the simulations, we conduct a strong scalability test, where the global problem size

\begin{tabular}{rrr}
\hline \#processes & total time $[\mathrm{s}]$ & efficiency \\
\hline 1 & 13975 & 1 \\
2 & 7763 & 0.90 \\
4 & 4151 & 0.84 \\
8 & 2658 & 0.65 \\
\hline
\end{tabular}

Table 5: Strong scalability test for test case 2

stays fixed and the number of processes is increased.

The efficiency is defined as

$$
E=\frac{T_{1}}{p T_{p}},
$$

where $T_{1}$ is the time for the sequential method, $p$ the number of processes and $T_{p}$ the time for the parallel method with $p$ processes. Table 5 shows the results for test case 2 for a simulation time of 65 days. The total time needed for solving the problem scales very well with the number of processes.

For a possible comparison with other implementations we list some important performance indicators in Table 6. TS is the amount of time steps that were performed (successful and unsuccessful), for average and minimum time step sizes only the successful time steps were regarded. NI is the average number of Newton iterations per time step (successful and unsuccessful), where $10 \mathrm{NI}$ are the maximum number of iterations that were allowed. Table 6 shows, that the average 


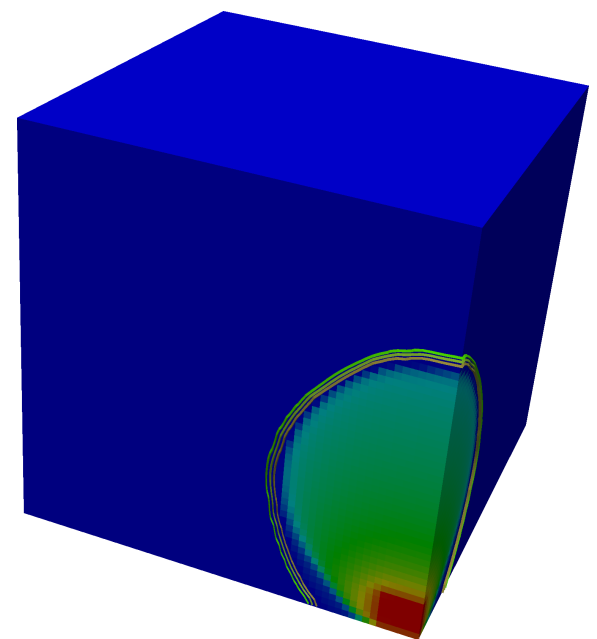

(a) 4 days,

$\max \left(S_{\mathrm{n}}\right)=0.65, \max \left(x_{\mathrm{w}}^{\mathrm{b}}\right)=0.021$

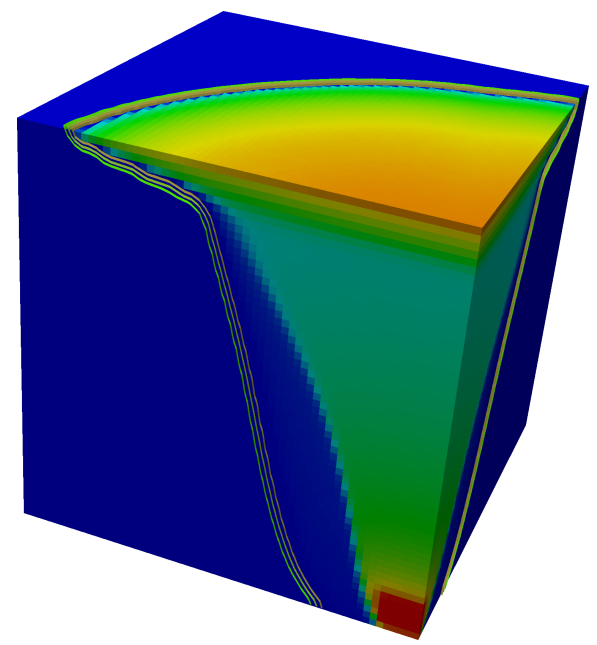

(c) 14 days $\max \left(S_{\mathrm{n}}\right)=0.82, \max \left(x_{\mathrm{w}}^{\mathrm{b}}\right)=0.021$

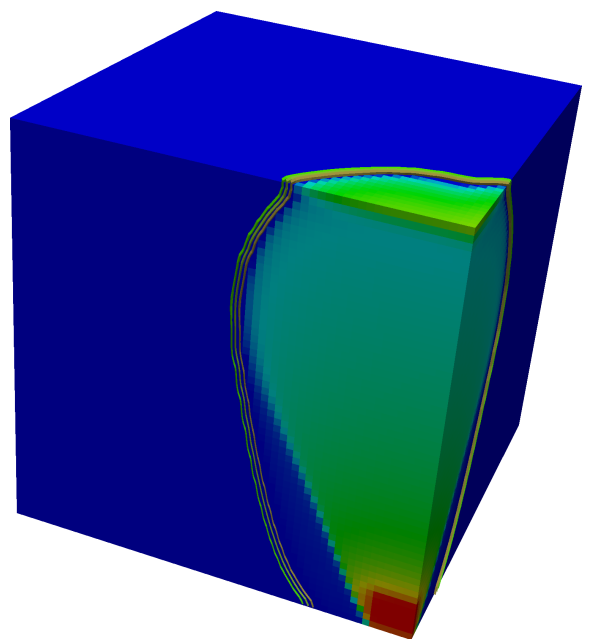

(b) 9 days, $\max \left(S_{\mathrm{n}}\right)=0.77, \max \left(x_{\mathrm{w}}^{\mathrm{b}}\right)=0.021$

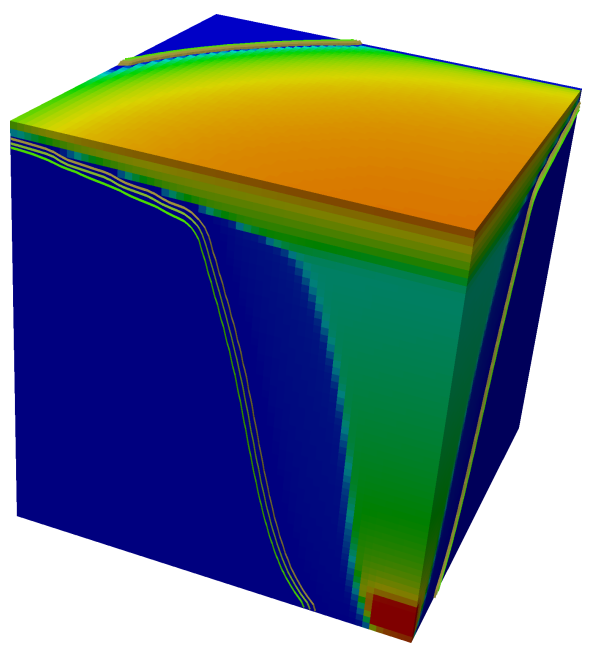

(d) 18 days, $\max \left(S_{\mathrm{n}}\right)=0.82, \max \left(x_{\mathrm{w}}^{\mathrm{b}}\right)=0.021$

Figure 10: Test case 3: $\mathrm{CO}_{2}$ phase saturation and molar fraction of dissolved $\mathrm{CO}_{2}$ in water (contour lines for $\left.x_{\mathrm{w}}^{\mathrm{b}}=0.005,0.010,0.016\right)$. Color scale ranges from $S_{\mathrm{n}}=0$ (blue) to $S_{\mathrm{n}}=\max \left(S_{\mathrm{n}}\right)$ (red).

\begin{tabular}{rrrrr}
\hline \#processes & TS & av. $d t$ & $\min . d t$ & av. NI \\
\hline 1 & 2249 & 3579.7 & 156.25 & 3.9 \\
2 & 2276 & 3527.6 & 156.25 & 3.9 \\
4 & 2205 & 3593.1 & 312.5 & 3.9 \\
8 & 2282 & 3514.4 & 312.5 & 3.9 \\
\hline
\end{tabular}

Table 6: Average time step size and number of Newton iterations of the strong scalability test for test case 2

time step size and number of Newton iterations stay almost constant for different number of processes.

\section{Test case 3: $\mathrm{CO}_{2}$ injection in a fully water saturated domain (3D)}

For test case 3, we use the same parameters and a very similar setup as in test case 2 in Section 7. The difference is that we look at a $3 \mathrm{D}$ domain as shown in Figure 11.

The domain is a cube with dimensions $100 \mathrm{~m} \times$ $100 \mathrm{~m} \times 100 \mathrm{~m}$. For the computations a structured grid with $60 \times 60 \times 60$ cells was used.

The results of test case 3 are shown in Figure 10 As in test case 2 each picture shows the $\mathrm{CO}_{2}$ phase saturation and the solubility of $\mathrm{CO}_{2}$ in the water phase. 


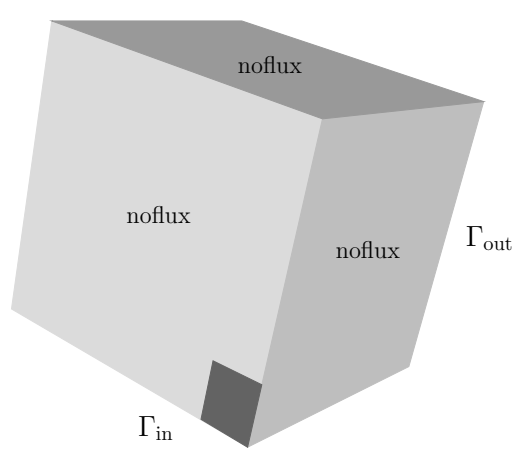

Figure 11: Test case 3: Domain setup

\section{Conclusion}

We suggest a new method to deal with the problem of disappearing nonwetting phase in two-phase twocomponent flow simulations. We use the nonwetting phase pressure and capillary pressure as primary variables. This allows us to use the same variables for both the monophasic and diphasic case, no switching of primary variables is needed to treat the nonwetting phase appearance problem. For the special case of CSS, we specify our choices for the necessary constitutive relations.

We confirm our new choice of primary variables with numerical simulations for different test cases in $2 \mathrm{D}$ and $3 \mathrm{D}$. We simulate the special case of $\mathrm{CO}_{2}$ injection in geological formations and took part in the MoMas benchmark on multiphase flow, where hydrogen flow in nuclear waste repositories was examined. All simulations are performed in parallel and scale very well with the number of processes. In the benchmark case our output corresponds very well to the results of other groups.

Next we want to extend our simulations to a nonisothermal model and use adaptive grid refinement. We want to use massive parallel computing in order to simulate realistic CSS scenarios with very large domains and long time spans.

Acknowledgments We would like to thank Holger Class, Lena Walter and Melanie Darcis from the Department of Hydromechanics and Modeling of Hydrosystems at the University of Stuttgart for many fruitful discussions regarding the properties of $\mathrm{CO}_{2}$-brine systems. This work was supported by the Baden-Württemberg Stiftung.

\section{References}

[1] Class, H.: Theorie und numerische Modellierung nichtisothermer Mehrphasenprozesse in NAPLkontaminierten porösen Medien. Ph.D. thesis, University of Stuttgart (2000)
[2] Bourgeat, A., Jurak, M., Smaï, F.: Modelling and Numerical Simulation of Gas Migration in a Nuclear Waste Repository. URL: http://arxiv.org/ abs/1006.2914 (2010)

[3] Spycher, N., Pruess, K.: CO2-H2O Mixtures in the Geological Sequestration of CO2. II. Partitioning in Chloride Brines at $12-100^{\circ} \mathrm{C}$ and up to 600 bar. Geochimica et Cosmochimicha Acta 69(13), 3309-3320 (2005)

[4] Duan, Z., Sun, R.: An improved model calculating $\mathrm{CO} 2$ solubility in pure water and aqueous $\mathrm{NaCl}$ solutions from 273 to $533 \mathrm{~K}$ and from 0 to 2000 bar. Chemical Geology 193, 257-271 (2003)

[5] García, J.E.: Density of Aqueous Solutions of CO2. Lawrence Berkeley National Laboratory LBNL49023 (2001)

[6] Duan, Z., Moller, N., Weare, J.H.: An equation of state for the $\mathrm{CH} 4-\mathrm{CO} 2-\mathrm{H} 2 \mathrm{O}$ system: I. Pure systems from 0 to $1000^{\circ} \mathrm{C}$ and 0 to 8000 bar. Geochimica et Cosmochimicha Acta 56(7), 2605-2617 (1992)

[7] Atkins, P.W.: Physikalische Chemie. VHC Verlagsgesellschaft (1990)

[8] Fenghour, A., Wakeham, W.A., Vesovic, V.: The Viscosity of Carbon Dioxide. Journal of Physical and Chemical Reference Data 27(1), 31-44 (1998)

[9] Jin, Y., Jury, W.A.: Characterizing the Dependence of Gas Diffusion Coefficient on Soil Properties. Soil Science Society of America Journal 60, 66-71 (1996)

[10] Abadpour, A., Panfilov, M.: Method of Negative Saturations for Modeling Two-phase Compositional Flow with Oversaturated Zones. Transport in Porous Media 79(2), 197-214 (2009)

[11] Jaffré, J., Sboui, A.: Henry's Law and Gas Phase Disappearance. Transport in Porous Media 82, 521-526 (2010)

[12] Forsyth, P.A., Simpson, R.B.: A two-phase twocomponent model for natural convection in a porous medium. International Journal for Numerical Methods in Fluids 12, 655-682 (1991)

[13] Class, H., Helmig, R., Bastian, P.: Numerical simulation of non-isothermal multiphase multicomponent processes in porous media. Advances in Water Resources 25(5), 533-550 (2002)

[14] Ippisch, O.: Coupled Transport in Natural Porous Media. Ph.D. thesis, University of Heidelberg (2003)

[15] Angelini, O., Chavant, C., Chénier, E., Eymard, R., Granet, S.: Finite volume approximation of a diffusiondissolution model and application to nuclear waste storage. Mathematics and Computers in Simulation 81, 2001-2017 (2011)

[16] Bastian, P., Blatt, M., Dedner, A., Engwer, C., Fahlke, J., Gräser, C., Klöfkorn, R., Nolte, M., Ohlberger, M., Sander, O.: DUNE Distributed and Unified Numerics Environment. URL: http://www.dune-project.org (2011) 
[17] Bastian, P., Blatt, M., Dedner, A., Engwer, C., Klöfkorn, R., Kornhuber, R., Ohlberger, M., Sander, O.: A Generic Grid Interface for Parallel and Adaptive Scientific Computing. Part II: Implementation and Tests in DUNE. Computing 82(2-3), 121-138 (2008)

[18] Blatt, M.: A Parallel Algebraic Multigrid Method for Elliptic Problems with Highly Discontinuous Coefficients. Ph.D. thesis, University of Heidelberg (2010)

[19] MoMaS Benchmark on Multiphase Flow in Porous Media - Exercise 1. URL: http://www.gdrmomas.org/ Benchmark/multi phase/multiphasique.html

[20] Bourgeat, A., Granet, S., Smaï, F.: Compositional Two-Phase Flow in Saturated-Unsaturated Porous Media: Benchmarks for Phase Appearance/Disappearance. Radon Series on Computational and Applied Mathematics : Simulation of Flow in Porous Media (2012). Submitted 